\title{
Review of Compressible Pulsating Flow Effects on System Performance
}

\author{
M. Metwally*
}

\begin{abstract}
Pulsating flow is as old as the human being. It appears in different forms from a compressible to incompressible, one phase, two or multi phase flows. Some forms of pulsating flow are favorable like that increase the combustion efficiency in combustors. Other forms of pulsating flow are harmful such as pulsation associated with compressor surge leading to increase of the noise level. Understanding the behavior of the pulsating flow in different forms, its effects on the flow system performance and on the gas turbine engine cooling system are reviewed. Reynolds number, velocity gradient, pressure gradient and frequency are the main parameters that affecting the pulsating flow behavior.
\end{abstract}

The measurement of pulsating flow in its forms is one of the major tasks of the researchers. From the review it appears that number of researches tried to cover the whole sides of pulsating flow phenomena and the different ways to measure it. However, a lot of works is still needed to be understood thoroughly the effect of pulsating flow on the flow systems and how it could be used to developed the performance.

Keywords: Pulsating Flow, compressible flow, unsteady flow, system performance, flow measurements.

\section{Introduction}

This introduction is to give an answer to the main questions related to the pulsating flow which are: What is meant by pulsating flow?, What are the different forms of pulsating flow?, What are the main parameters that affecting pulsating flow?, How could the pulsating flow affect the flow system performance?, What is the necessity of studying pulsating flow?

Pulsating flow is a particular kind of unsteady flow in which there is a regular cyclic variation in the flow velocity superimposed on a constant time-average flow rate. In most industrial pipe flows the nature of the flow is turbulence and when pulsations are also present there will be both random turbulent fluctuations and cyclic variations in velocity. The nature of typical pulsating flow has changed from low frequency, high amplitude fluctuations to high frequency flow pulsations of relatively low amplitude.

The pulsating flow is generated in a number of practical situations, such as:

- Eddy turbulence; turbulence associated with a so-called steady flow. It is in fact a random and multidirectional.

- Flow in turbomachinery; pressure and velocity gradients, established in the space between adjacent blades in a rotating component (turbines and compressors).

\footnotetext{
*Egyptian Armed Forces, m2metwally@yahoo.com
} 
- Flow in natural-gas pipelines during transient flow where changes in operating conditions are applied.

- Flow in inlet and exhaust systems of reciprocating internal combustion engines.

- Pulsating flow appears when the gas turbine engine encounters the surge zone. The surge phenomenon is defined as an axisymmetric oscillating flow through the gas turbine engine. Compressor surge of gas turbine engine is the most unwanted and drastic form of pulsating flow.

- Pulsating flow in the displacement pump.

- Breath in the human being etc........

On the other hand, not all the forms of pulsating flow are unwanted and drastic. There is a pulsating flow used to enhance the combustion performance and to get a complete mixing between the fuel and air in the combustor. Numbers of researches have been studied theoretically and experimentally the behavior of the compressible pulsating flow behavior and its effect on the combustion performance $[5,11,12,13,18,19]$. Another researchers have investigated the behavior of two phase pulsating flow such as in the manifold prior the combustion chamber [13].

Notable among the advantages of pulsating flow in combustion process are:

- High combustion efficiency.

- Ability to burn different types of fuel with high combustion efficiencies, while utilizing low values of excess air.

- High convective heat transfer rates.

- Reduced pollutants formation.

- Capability for self aspiration.

Beside the effects of pulsating flow in enhancement the combustion process, pulsating flow has a great effect in studying the stability of pipes and fittings. A phenomenon that appear adhere to the pulsating flow like the noise of exhaust either from the reciprocating engine or from gas turbine engine in an unsteady conditions has also attracted a number of researchers $[11,15]$ to find out their effect of the flow and way to reduce it.

There are researches describing the difficulties of observing accurate flow behavior and measurements in the presence of pulsation dating back to the 1920s [1]. As might be expected, the main affecting parameters on the pulsating flow behavior are the Reynolds number, velocity gradient, pressure gradient and frequency.

This paper will be concerned primarily on the measurement of compressible pulsating flow. The effect of pulsating flow in the enhancement of the flow system performance has been introduced. The effect of pulsating flow in the noise generating from the system has been also reviewed.

\section{Measurement of Compressible Pulsating Flow}

The problem with pulsating flow parameter is that there is no simple means for measuring it. Contrary to the steady flow, the distributions of mean-velocity, pulsating velocity fluctuations and turbulence velocity fluctuations are not uniform across the pipe cross-section. This is illustrated in figures ( 1 and 2$)$, which show both time mean and rms velocity distributions 
measured by hotwire traverses across a pipe diameter in pulsating air flow. Figure (1) shows distribution at $5 \mathrm{~Hz}$, whereas figure (2) shows similar plots at $19 \mathrm{~Hz}$. The measurement of $\left(\mathrm{U}_{\mathrm{rms}} / \mathrm{U}\right)$ on the pipe centerline multiplied by a factor could be used to represent $\left(\mathrm{U}_{\mathrm{rms}} / \mathrm{U}\right)$ for the bulk flow; where: $U_{\text {rms. }}$ is the root mean square of the variations above and below the time mean of the bulk-mean velocity, $\mathrm{U}$; and $\overline{\mathrm{U}}$ is the average velocity.

The plots on Figures (1 and 2) clearly show that the factor does not have a constant value. Unfortunately the multiplying factor is itself a variable, depending on the pulsating flow frequency, amplitude waveform and possibly the acoustic characteristics of the pipe-work. Thus a single hot-wire measurement can only give an approximate indication of the velocity pulsation amplitude $[4,7,16]$.

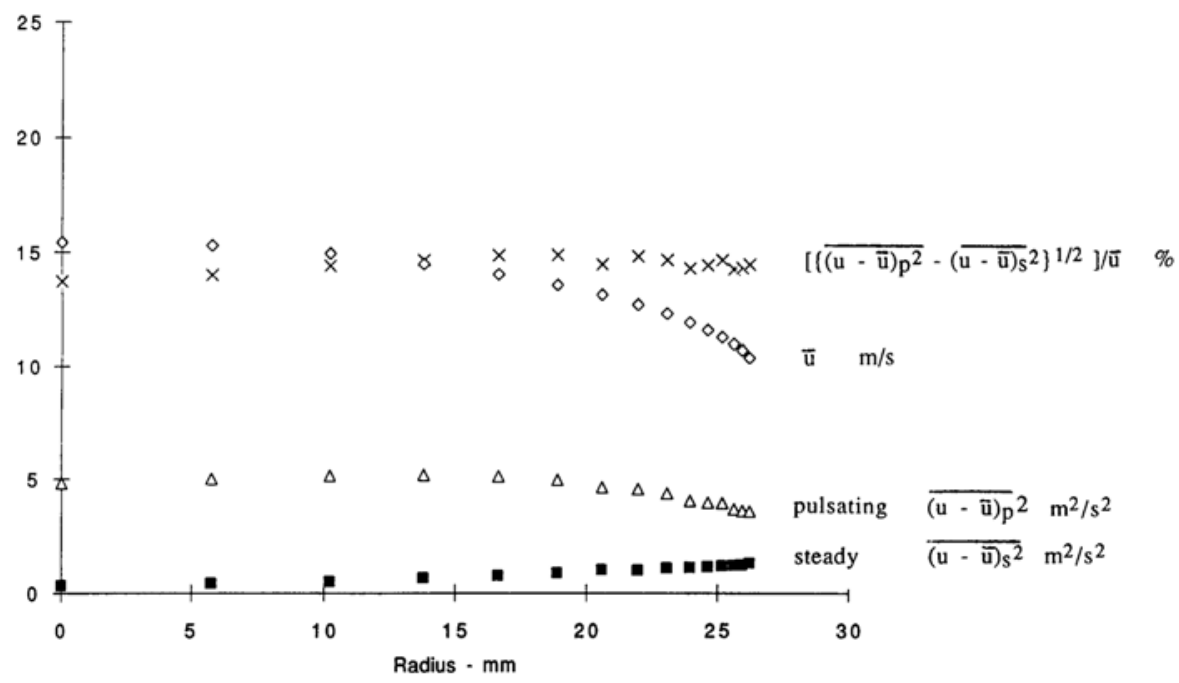

Fig. 1 Velocity profiles measured in a $54 \mathrm{~mm}$ bore pipe under steady, fully developed turbulent flow conditions and pulsation frequency of $5 \mathrm{~Hz}$ [7]

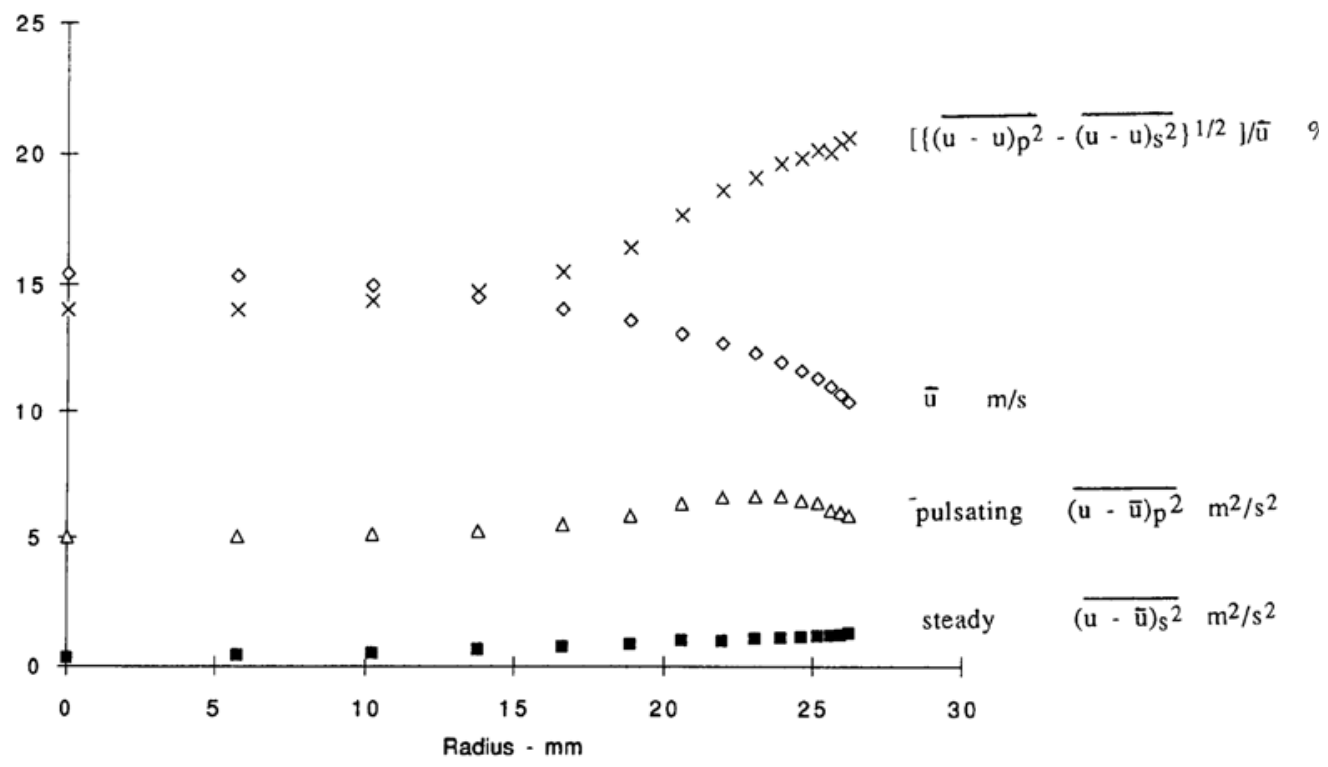

Fig. 2 Velocity profiles measured in a $54 \mathrm{~mm}$ bore pipe under steady, fully developed turbulent flow conditions and pulsation frequency of $19 \mathrm{~Hz}$ [7] 
As might be expected, the amplitude of the velocity pulsations is an important parameter in the characterization of pulsation effects on flowmeters. This is particularly true with turbine meters [9] and also differential pressure type flowmeters such as the orifice plate [1]. The parameter which appears in the theoretical expressions for metering error for both orifice plates and turbine meters is the amplitude ratio $\mathrm{U}_{\mathrm{rms}} / \mathrm{U}[1,7,10,16]$

A device such as a hot-wire probe can only respond to local velocity [4]. Recently, one promising principle with the fastest growing market share lies in ultrasonic devices which sending ultrasonic beams through the fluid. The changed properties of ultrasound such as time-of-flight or the frequency- shift refer to the speed of the fluid [19].

Orifice plate meter is a commonly used device for the measurement of air flow. Any discussion on orifice plate behavior in pulsating flow is normally divided into two parts:

- The relationship between flow rate and the differential pressure across the orifice.

- The accurate measurement of the differential pressure.

Existing standards for flow measurement specify that the fluctuations of the flow must be maintained at a low value and should not exceed $10 \%$ of the mean flow for reasonable accuracy results to be obtained from an orifice plate meter. For higher values of flow fluctuations the recommended practice is to add a reservoir between the flow-meter and the source of pulsations [1]. With the aim of better defining the correction factors to be used for the measurement of pulsating flow with orifice plate meters, One-dimensional flow model could be used to evaluate the coefficient of discharge $(\tau)$ and the coefficient of pressure drop across an orifice plate in a pulsating flow of air.

$$
\tau=\frac{\text { maximum flow }- \text { min imum flow }}{\text { maximum flow }+ \text { min imum flow }}
$$

It is found that the coefficient of pressure drop continues to have the same value as in steady flow but the coefficient of discharge is modified. These coefficients are evaluated by assigning them such values, which when introduced in a simplified one dimensional mathematical model of the experimental set up would give a reasonable identification with the measured values, figure (3). It is shown that the effect of introducing an orifice plate into a pipe is to increase the apparent coefficient of friction applicable to the fluctuating component of velocity. Also the coefficient of pressure drop across the orifice plate has the same value as in the case of steady flow. The coefficient of discharge of an orifice plate in pulsating flow is modified and as a result the flow indicated by an orifice meter would be higher.

Square root errors of pulsating flow measurements by pressure differential can only be avoided by measuring or computing the average of the square roots of signals from a fast response differential pressure transducer. Another major source of errors in pulsating flow is due to the long distance separated the differential pressure sensor from the pressure tappings often by several meters of tubing and numerous fittings and isolating valves etc. Acoustic effects can cause severe distortion in the pressure waves and as a consequence, large errors in the indicated differential pressure are resulted. A third major source of pulsation induced errors in orifice plate flowmeters is the sequential inertia effect. This effect is due to the fact that a component of the differential pressure is required to accelerate the fluid with respect to time in addition to that required for the convective acceleration through the orifice [10]. 


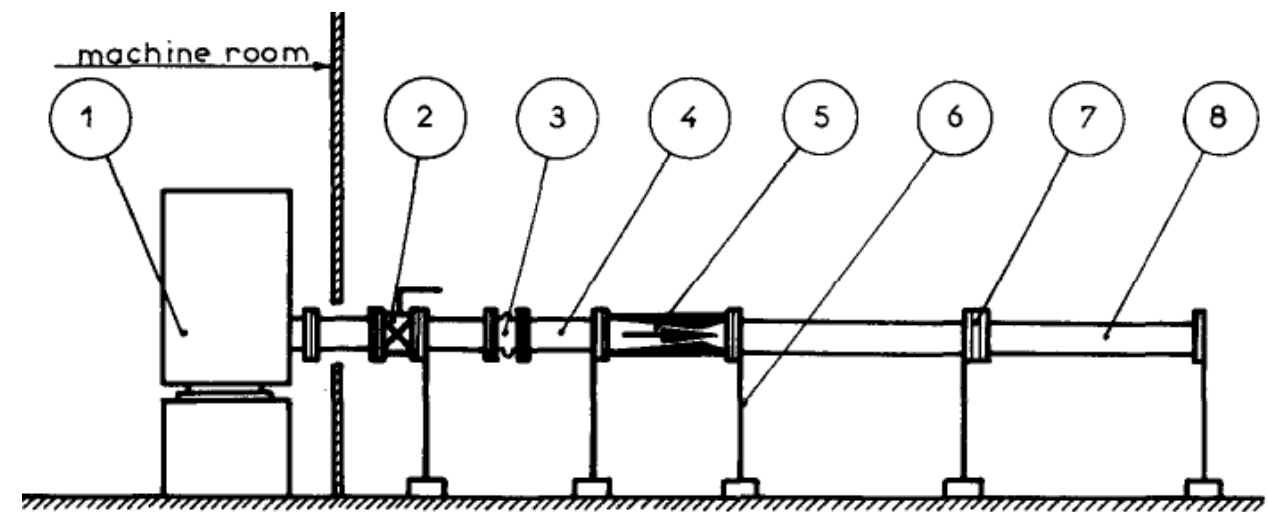

1- Water ring vacuum pump ; 2- Butterfly valve ; 3- Antivibration joint 4- Diffuser ; 5- Convergent-divergent nozzle and reciprocating body 6- Supports ; 7- Orifice plate ; 8- P.V.C pipe $104 \mathrm{~mm}$ diameter

Fig.3 Orifice plate Experimental set-up. [1]

The error will depend on the relative amplitude of flow pulsations. The reasoning given in this paper indicates that the mean indicated flow will be higher by $1.5 \%$ for flow pulsations of $6 \%$ and $4 \%$ higher for flow pulsations of $15 \%$. The coefficient of discharge of an orifice plate in pulsating flow is modified and as a result the flow indicated by an orifice meter would be higher. If the pulsation frequency is more than about $2 \mathrm{~Hz}$ most industrial flowmeters, differential pressure and temperature sensors will be too heavily damped to respond. Thus the presence of flow pulsation may easily go undetected $[1,7,10]$.

Connecting a fast response differential pressure transducer across the tappings of an orifice plate or other differential-pressure-generating device and inspecting the time-trace or the frequency spectrum of the signal is another possibility. Resonance in the connecting leads, however, can produce confusing effects when the lead lengths are not short compared with the quarter-wavelength of any pulsation present. There are numerous difficulties in measuring pulsating differential pressures. The pulsation error due to the basic non-linearity of the pressure differential flow meter arises when a slow-response differential sensor is used. In this case the signal generated is proportional to a time-mean differential pressure and the square root of this will always give an indicated flow rate greater than the true value. Unless the amplitude of the velocity pulsation is known, it is not possible to correct the indicated value. A theoretical solution to this problem is to use a fast-response transducer to allow the flow rate to be computed from the instantaneous square-root differential pressure signal. It describes drawbacks to be avoided if accurate dynamic differential pressure measurement is required $[10,16]$.

Pulsation-damping criteria have always been associated with differential-pressure type flowmeters $[1,7]$. The mathematical form of the criteria, however, makes it suitable for use with any flowmeter for which there is a relationship between metering error and flow pulsation amplitude. The use of this form of damping and the development of the associated criteria for adequate damping were pioneered by Hodgson in the 1920s [7]. He was attempting to solve the obvious pulsation problem which arose when differential-pressure type flowmeters were used to measure pulsating flows. The pulsation amplitude at the flow meter is reduced when a sufficiently large receiver is introduced into the system between the meter and the source of pulsation, as shown in figure (4). The effectiveness of the damping 
increases with the volumetric capacity of the receiver and the overall pressure loss between the pulsation source and the far side of the flowmeter primary element, i.e. the orifice plate, nozzle or venture tube. The pressure loss can be increased by reducing the area ratio of the primary element or by introducing additional throttling in the form of a valve. Hodgson demonstrated that a criterion could be defined in terms Hodgson number, $\mathrm{H}_{0}$, formulated as:

$H_{o}=\frac{V}{\bar{q}_{v} / f} \frac{\overline{\Delta p}}{\bar{p}}$

where

$\mathrm{V} \quad$ Volume of the receiver between pulsation source and flowmeter

$\overline{\mathrm{q}}_{v} \ldots \quad$ Time-mean volumetric flowrate at the mean density in the receiver.

$\bar{p} \ldots \quad$ Time-mean absolute static pressure in the receiver.

$\overline{\Delta \mathrm{p}} \ldots \quad$ Overall time-mean pressure loss between the receiver and the source of supply (or discharge) at constant pressure,

f ...... Pulsation fundamental frequency.

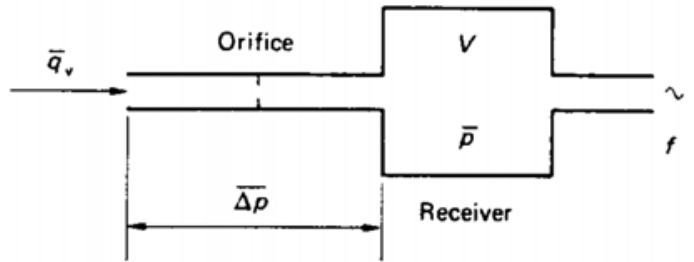

a

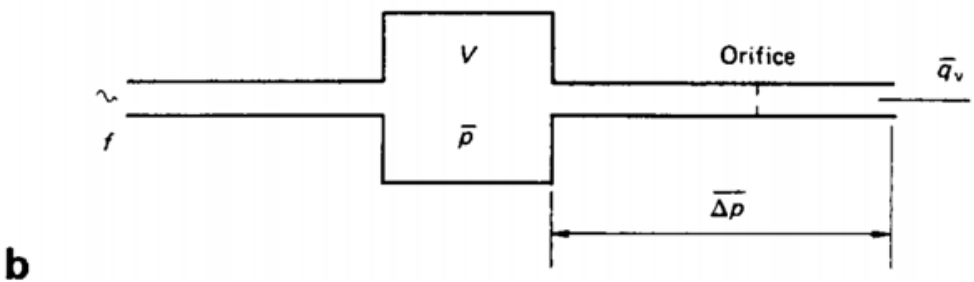

Fig. 4 Single-receiver damping system [7].

(a) Pulsation source downstream of meter, (b) Pulsation source upstream of meter

The error in the indicated flowrate of a differential-pressure type flowmeter can be related to the velocity pulsation amplitude ratio by the Equation (3) [7]:

$E_{T}=\left[1+\left(\frac{U_{r m s}}{U}\right)^{2}\right]^{1 / 2}-1 U$ 


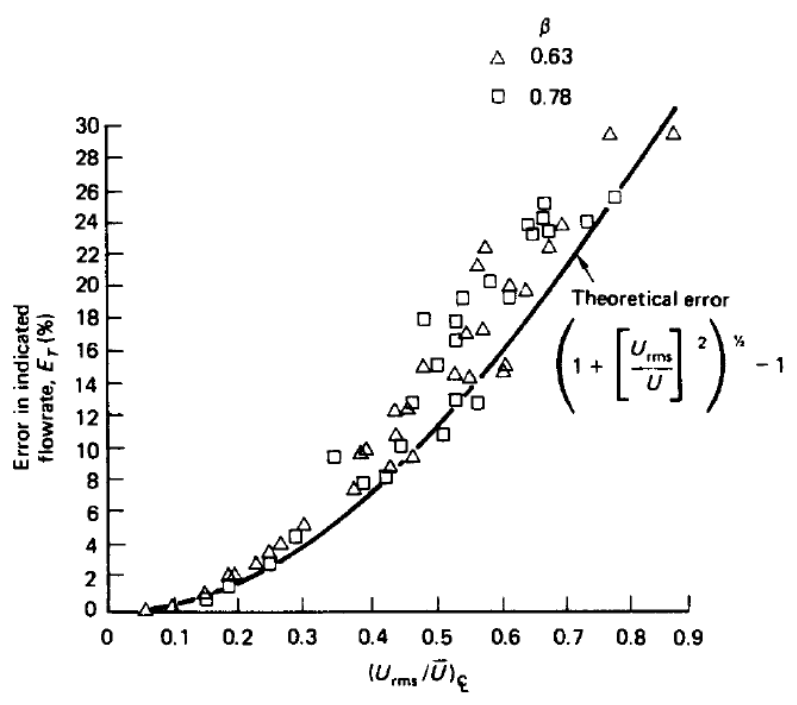

Fig. 5 Correlation of pulsation errors in orifice meters with velocity pulsation amplitude [7]

Figure (5) shows results obtained at the University of Surrey on an air flow rig on orifice plates with corner tappings using a special differential-pressure sensor with very short connecting leads to eliminate secondary system errors due to pressure wave distortion [7]. The pipe bore was $80 \mathrm{~mm}$ and the pulsation frequency range was $0-50 \mathrm{~Hz}$. It can be seen that the correlation of experimental results with the theoretical relationship of Equation (3) is quite good when the amplitude ratio is less than 0.13 . This corresponds to metering errors of less than $4 \%$. This good correlation at low pulsation amplitudes is particularly significant as we are interested in the conditions after damping.

If pulsations are suspected, however, there are a number of techniques that can be used to detect them and possibly to assess their severity. Turbine meters are essentially instruments for metering steady flows it is often argued that their response makes them suitable for metering flows in which a degree of unsteadiness is present. Turbine flowmeters are now much used in the economic metering of both liquids and gases. For this reason their behavior in non-ideal flow conditions is of great interest. The fact that they tend to over-register under pulsating flow conditions is now well known [7,9] and is of great concern to their users. If the over-registration can be predicted from pulsation parameters that can be easily measured at an industrial meter installation the meter error can be corrected. Analysis of the equation of motion for a single rotor turbine meter under pulsating flow conditions shows that the amplitude of the velocity pulsations and the pulsation frequency are both important parameters affecting meter error. Unfortunately the velocity pulsation amplitude is not a quantity that can be conveniently determined by direct measurement outside of a flow laboratory. If it can be inferred from some other measurement, say of dynamic pressure or even from the turbine meter signal itself, this might make it feasible to correct the pulsation error. The response of the meter is proportional to the density of the fluid, so in general the meter is able to follow any unsteadiness in liquid flows than in gas flows. If high frequency pulsations are present, particularly in gas flows, then the meter lack of response will cause it to over-register the mean flow rate. This happens because the meter responds more rapidly to the increase in flow rate rather than to the decrease. The size of the over-registration error depends upon, among other things, the frequency and amplitude of the pulsations and the inertia of the rotor. The error increases with the frequency and reaches a constant value when 
the frequency is too high that the rotor turns at constant speed. The over-registration error of a turbine meter solely on the basis of the meter signal has been calculated by software. The developed software has calculated the over-registration errors by introducing the root main square of the flow rate amplitude and the flow meter response depends on flow properties and rotor inertia and aerodynamic characteristics [9]. The software tool is based upon the differential equation of motion of the meter, which is given by:

$b \frac{d f}{d t}=q^{2}-q f$

where:

$\mathrm{f} \quad$ instantaneous volume flow rate as given by the meter $(=\mathrm{k} \omega, \mathrm{k}$ is a constant and $\omega$ is the angular velocity of the rotor).

q actual instantaneous flow rate.

b parameter determines how quickly the meter responds to the changes in the flow rate.

The software has been using a pure sinusoidal wave as an assumption of the inlet flow wave form. Experimental setup to test the accuracy of the software tool is shown in figure (6).

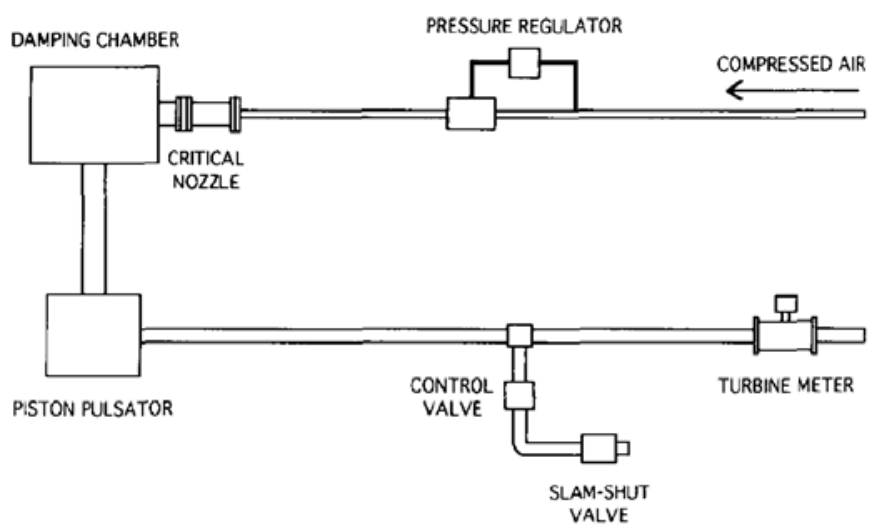

Fig. 6. Turbine meter experimental setup [9]

In the experiment input parameters, the author [9] didn't use a pure sinusoidal wave in the inlet flow rate, the usage of a pulsating wave and a step input was introduced in-stead. The frequency and amplitude of the pulsations have been measured by hot-wire probe. The pulsation generator was a variable-stroke piston displacement compressor which had a (2-50) $\mathrm{Hz}$ frequency range and could generate pulsation amplitudes up to $100 \%$. Figure (7) shows a typical hotwire trace of the pulsations, indicating that the waveform is near-sinusoidal.

To investigate the step response of the meter a solenoid-operated slam-shut valve has been positioned upstream of the meter. Shutting the valve produced a step increase in the flow rate through the meter. 


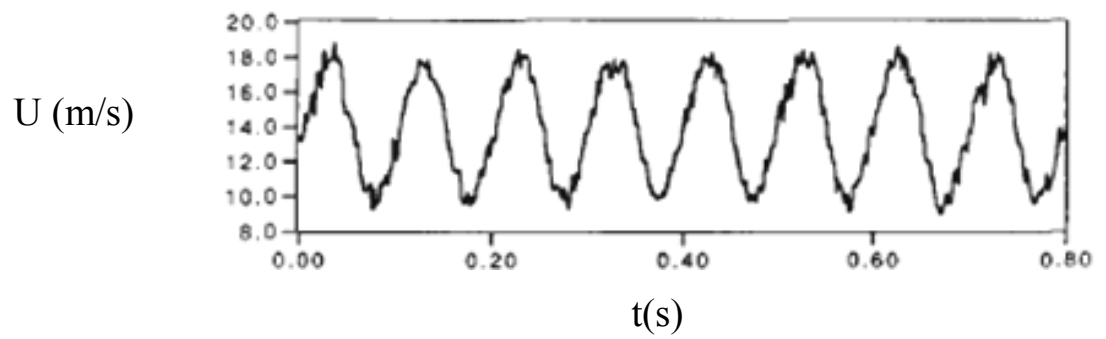

Fig. 7 Hot-wire trace of the flow pulsations for actual mean flow rate $=0.026 \mathrm{~m}^{3} / \mathrm{s}$, $\mathbf{t}=\mathbf{0 . I} \mathrm{s}$ and $\alpha_{r m s}=0.25[9]$

The measured and computed meter error values are plotted against B (Dimensionless form of meter response parameter) in figure (8). There is very close agreement throughout the range of $\mathrm{B}$ and $\alpha_{r m s}$ (Dimensionless root mean square of fluctuation in $\mathrm{q}$ about $\mathrm{q}_{\mathrm{m}}$ over period $\mathrm{T}$ ). The fact that the meter error values collapse onto the same line within the overlap region is proof that the frequency and flow rate can be grouped into the dimensionless parameter B. Also shown in figure (8) is the theoretical meter error for the average amplitude computed by the software tool over each test series.

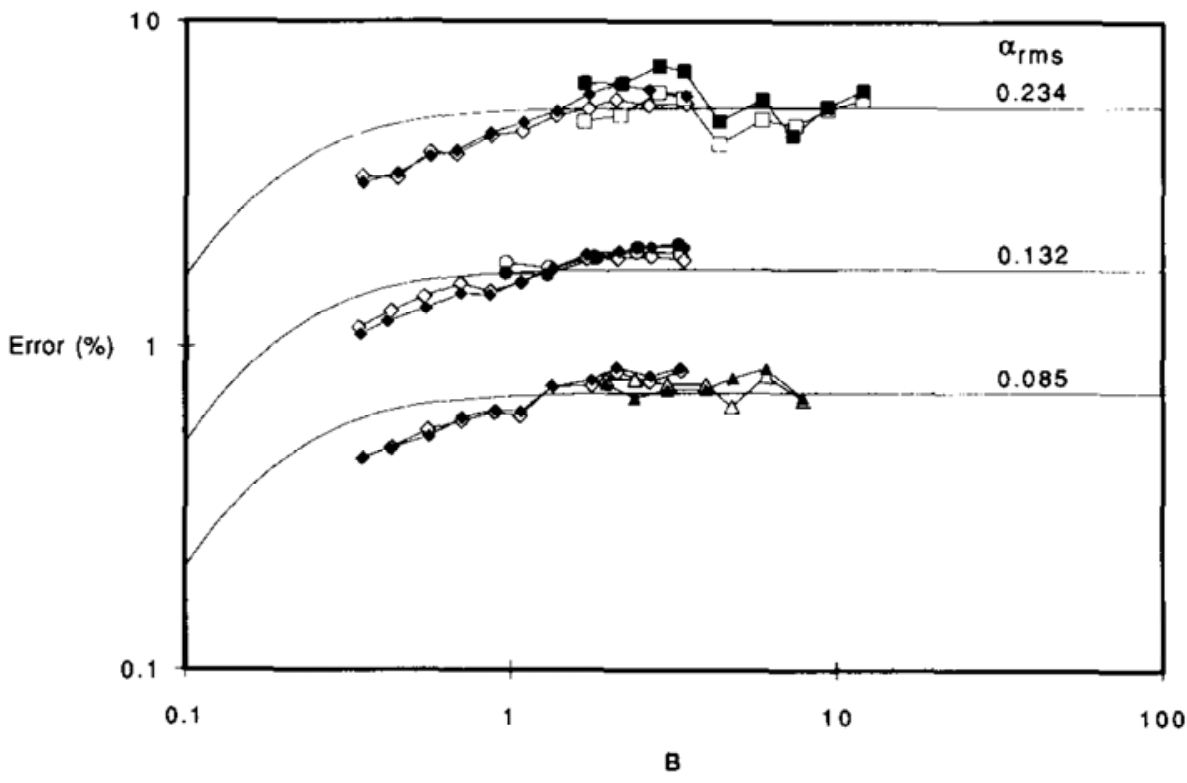

Fig. 8 Calculated and measured mean meter error [9]

Solid symbols, calculated; open symbols, measured; $\diamond \diamond q_{m}=0.0266 \mathrm{~m}^{3} / \mathrm{s} ; \boldsymbol{\Delta}, \triangle 0.0118 \mathrm{~m}^{3} / \mathrm{s} ; \boldsymbol{O}, \bigcirc$ $0.0090 \mathrm{~m}^{3} / \mathrm{s} ; \mathbf{\square}, \square 0.0059 \mathrm{~m}^{3} / \mathrm{s}$; — theoretical error for average value of calculated $\alpha_{\text {rms }}$

It should be possible to develop a Hodgson-type damping criterion for any flowmeter for which there is a relationship between metering error and velocity pulsation amplitude. Such a relationship exists for turbine meters operating at pulsation frequencies that are high compared with the rotor response frequency. During a cyclic variation in flow, the turbine 
rotor is better able to accelerate as flow increases than decelerate when flow decreases. The result is that the meter tends to read high in pulsating flow. The magnitude of the metering error increases with frequency, reaching a maximum value which is a function of the pulsation amplitude and waveform. For a sinusoidal waveform of flow amplitude about a mean value, the maximum metering error can be equivalent to $\left(\mathrm{U}_{\mathrm{rms}} / \mathrm{U}\right)^{2}$ and compares with the theoretical pulsation error for a differential pressure flowmeter of $0.5\left(\mathrm{U}_{\mathrm{rms}} / \mathrm{U}\right)^{2}$ for low amplitude pulsations, i.e. the maximum pulsation error in a turbine meter is twice that produced in an orifice meter for the same amplitude pulsation [7].

Beside the usage of turbine meter to measure of pulsating flow and in order to control precisely the air-fuel ratio in automotive engines, it is necessary to measure the intake air mass exactly. Hot-wire anemometry is a well-established method for measuring mass flow. This method has been applied experimentally for some time to measuring intake air mass in automobiles. A hot-wire air flow sensor has the following excellent points for an engine control system. First, air mass flow can be measured directly, which means that the sensors for compensating density variations can be eliminated. Secondly, we can expect very accurate measurements over a wide air flow range, because of its original non-linear characteristics. The response time is sufficiently fast to trace intake pulsation. It also has other features, such as no moving parts, low cost potential and easy mounting. A developed hot-wire air flow meter for automotive was designed to measure the pulsating flow rate as shown in equation (5) [4].

$Q=\left(C_{1}+C_{2} \sqrt{\sigma u}\right)\left(T_{w}-T_{a}\right) S$

An electronic control circuit for a hot-wire sensor has been fabricated on an alumina substrate using thick film technology, and the sensing probes have been connected to this substrate directly with the rod electrodes. Thus, the electronic circuit, as shown in figure (7), and sensing probe have been integrated in a small area. The specifications of the hot-wire air flow meter are as follows. Measuring range is typically 9 to $360 \mathrm{~kg} / \mathrm{h}$. The deviation of the measured mass air flow value is less than $4 \%$ of the indicated value, when the dynamic range is full scale/40 to full scale and ambient temperatures from 25 to 55 (G Pressure drop $\mathrm{m}$ the sensor assembly $1 \mathrm{~s}$ less than $2 \mathrm{kpa}$ The fully assembled sensor was tested in severe automotive environmental conditions, and It became clear that the maximum deviation of only $3 \%$ from indicated values was observed during severe durability tests.

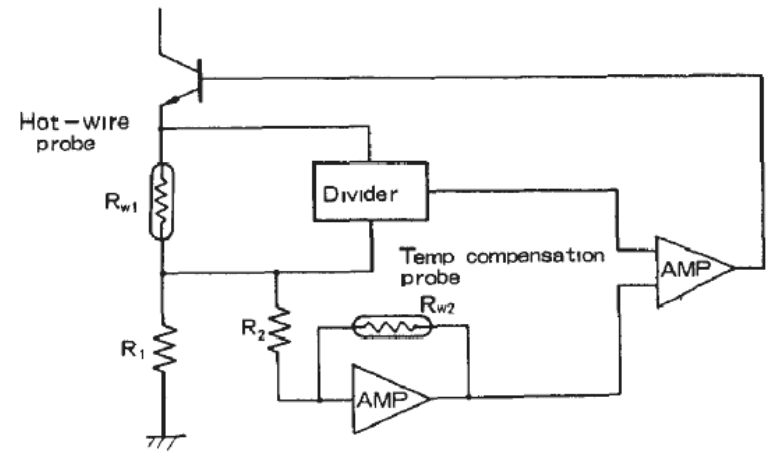

Fig. 9 The principles of the hot-wire control circuit. [4]

There are also meter types, however, which are often assumed to be unaffected by pulsations. This is the case for the electromagnetic flowmeter, the ultrasonic time-of-flight meter, and the Coriolis mass flowmeter types. Experimental work on the behavior of these meters in pulsating flow is long overdue. 
The ultrasound gains an increased acceptance due to its minor feedback to physical objects. Although destructive effects of ultrasound are well known, they usually depend on the intensity of the beam which can be controlled within wide limits as well in its amplitude as in its frequency. In the first instance two common principles for ultrasound measurements are described which help to understand the general measurement technique [16, 19]. Dopplerdevices send an ultrasonic beam through the fluid which is reflected at particles moving downstream. Due to the Doppler-effect, the reflected signal has a different frequency according to the amount of the speed component of the fluid in the direction of the ultrasonic beam. Two main measurements could be obtained by the ultrasound the cross correlation flow meter and the vortex flow meter. In the cross correlation flow meter, figure (10), was used for determining the flow rate of multi-phase flow. The maximum of a cross correlation function between the signals indicates the time shift $(\tau)$. With a known distance (d) between the barriers the mean velocity is determined by equation (6) [16].

$U=\frac{d}{\tau}$

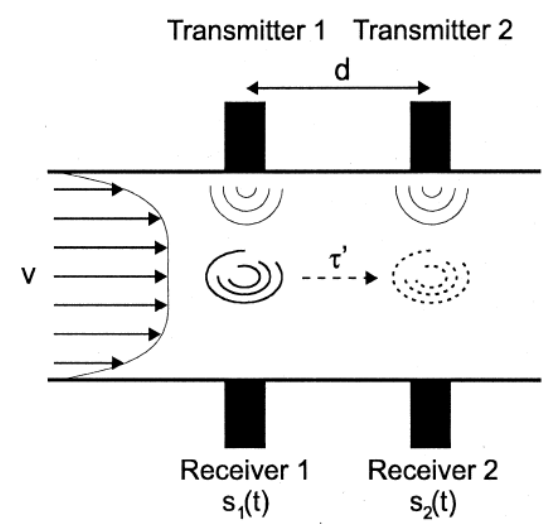

Fig. 10 Principle of cross correlation flow meter[19]

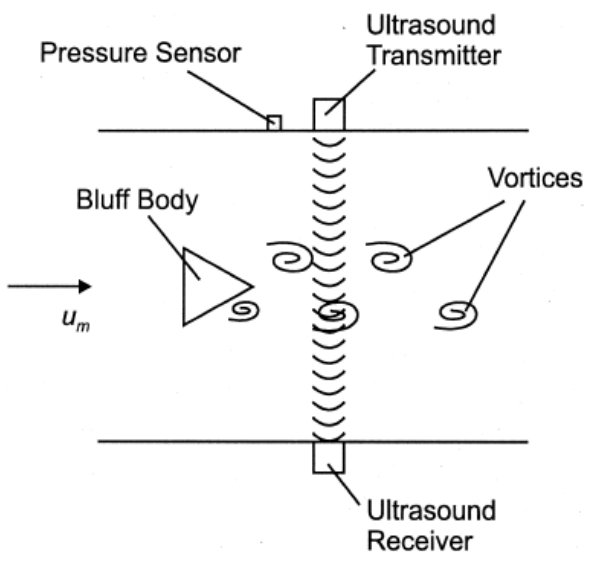

Fig. 11 Principle of Vortex shedding flow meter[19]

Vortex flow meters, figure (11), use the separation of vortices at the backside of a body that is flowed around. This phenomenon is well known as the Karman vortex street. The bluff-body pervades the pipe perpendicular to the axis. The dependence of the vortex frequency $f$, the 
width of the bluff body $\mathrm{d}$ and the velocity $\mathrm{u}$ are described by the dimensionless Strouhal number $[16,19]$ :

$$
S t=\frac{f \cdot d}{U}
$$

Flow field in and from the accumulator is a further form of pulsating flow. There are little of researches published examining the flow fields inside the accumulator under real working conditions. The flow fields in a proto-type accumulator operating under real working conditions were investigated experimentally using the hybrid particle tracking velocimetry (PTV) velocity field measurement technique. These results can be used not only to guide the design of a high performance accumulator but also to validate numerical predictions [21].

The flow inside the accumulator was qualitatively visualized using a high speed camera. The period of one cycle of the rotary compressor was divided into 4 phases and the velocity fields inside the accumulator were measured using a phase-averaging technique. The flow inside the accumulator, especially in the region between the screen holder and tube holder, showed a pulsating periodic flow structure according to the rotational phase of rotary compressor.

There are clearly many types of flowmeter which are not covered in the above review. Some meter types such as cone-and-float meters are known to suffer serious errors when subjected to pulsating flow. Some types, such as those using fluidic oscillators, are suspected of being sensitive to flow pulsations, although as yet there is nothing published in the literature. Fluidic oscillators belong to the same family as vortex-shedding flowmeters and are thus likely to be susceptible to lock-in.

\section{Effect of Compressible Pulsating Flow in Flow System Performance:}

The effect of pulsating flow on the flow system performance is reviewed, from point of view of its effect on combustion and flow branching stability. The interest in pulsating flow in combustion process has been increasing in recent years because of the phenomenon offers definite advantages in processes involving energy production. Notable among the advantages are $[3,5,13]$ :

- High combustion intensities.

- Ability to burn solid fuels with high combustion efficiencies while utilizing low values of excess air.

- High convective heat transfer rates.

- Reduced pollutants formation.

- Capability for self aspiration.

Few investigations have dealt with the interaction between particles and a pulsating flow. Research in this area can provide important information on the effects of oscillations on parameters such as; burning, heat transfer and pollutant formation rates, and particle trajectories. The burning time and the formation of ashes attract a number of researchers to improve the performance of the flow system by improving the combustion properties inside the combustors. The pulsating flow appears to reduce the burning times of individual coal particles, and the ratio of the burning time in oscillating flow to that in still air is a monotonically decreasing function of the ratio of the displacement amplitude of the gas to the radius of the particle $\left(\varepsilon=\mathrm{U}_{\max } / \omega \mathrm{R}_{\mathrm{i}}\right)$ [3]. The burning times of captive coal particles in oscillating flow of controlled velocity amplitude and frequency have been measured. This 
study was undertaken to explain the high values of combustion intensities reported in experiments with pulsating combustors. The experimental apparatus has been designed to measure the burning times of captive coal particles in oscillating flow of controlled frequency and velocity amplitude. The oscillating flow was set up in the neck of a Helmholtz resonator [3]. The Helmholtz resonator was used because it has a sharp resonance at its fundamental frequency, with a large magnification of the velocity amplitude The experimental data, presented has been indicated that higher values of the ratio of the air displacement amplitude to the initial radius of the coal particle result in greater reduction in the burning time. The reduction in burning time varies from about $50 \%$ for a $1.3 \mathrm{~mm}$ diameter particle with a flow frequency of $54 \mathrm{~Hz}$ to about $10 \%$ for a $2.4 \mathrm{~mm}$ diameter particle with a flow frequency of 130 Hz. Oscillating flow seems to reduce the burning time, but the effect is less significant for larger-sized particles. Also, the reduction in burning time caused by low-frequency oscillations seems to be more pronounced than that caused by flow with higher frequency as shown in figure (12).

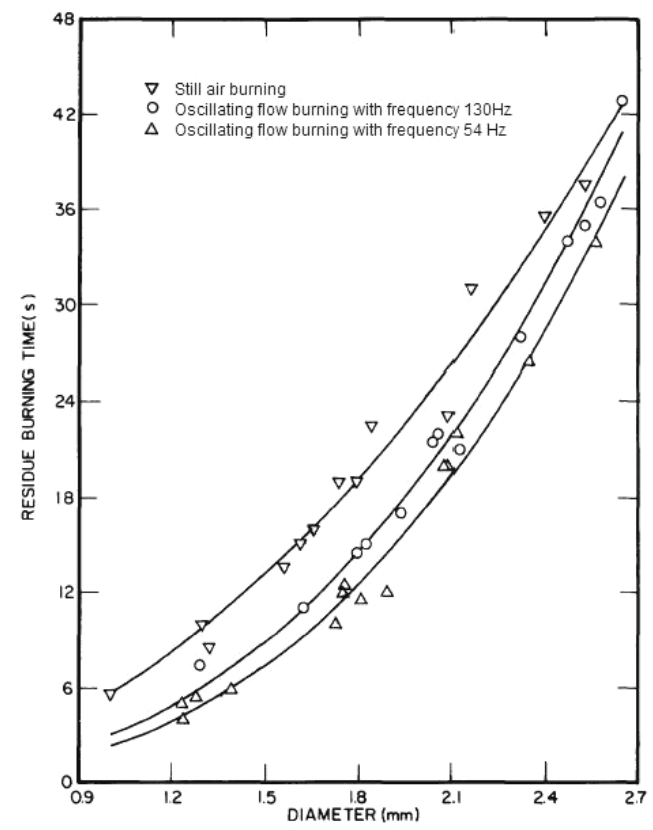

Fig. 12 Residue burning time vs. initial particle diameter, with and without oscillating flow. [3]

Transition from a non-pulsating to a pulsating operation in a Rijke type combustor results in increased combustion efficiency, increased combustion intensity, increased convective heat transfer to the combustor wall and decreased production of particulates. These data strongly suggest that the presence of pulsations may considerably improve the performance of combustors. an experimental investigation has been described the differences observed when a Rijke type coal combustor was operated under pulsating and non-pulsating conditions [5]. Non-pulsating operation was achieved by opening a small hole in the combustor's wall a few inches above the combustion bed. It was verified that the presence of acoustic oscillations results in significant improvements in the combustor performance. It has been observed that a transition from a non-pulsating to a pulsating operation in a Rijke type combustor results in increased combustion efficiency, increased combustion intensity, increased convective heat transfer to the combustor wall and decreased production of particulates as shown in figure (13). 


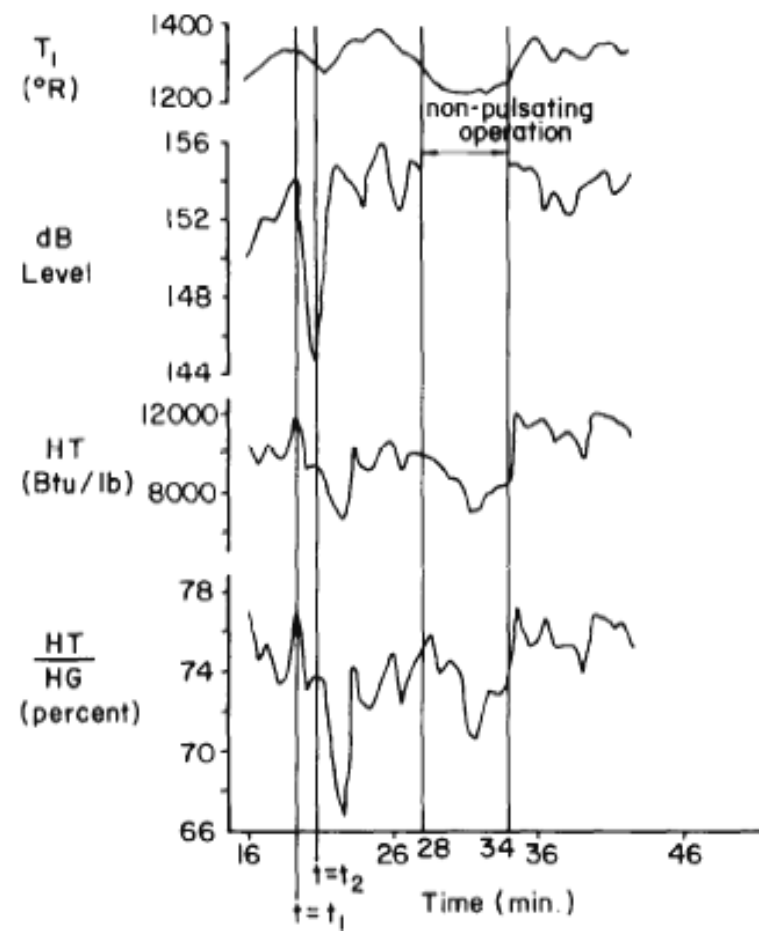

Fig. 13 Time variation of the flow gas temperature $\left(T_{1}\right)$, sound $d B$ level, heat transfer to the wall $\left(H_{T}\right)$, and the percentage of generated heat transferred to the wall $\left(H_{T} / H_{G}\right)$. [5]

For example, switching from non-pulsating to pulsating operation was accompanied by an increase of approximately $20 \%$; in the instantaneous combustion efficiency and a decrease of approximately $58 \%$, in the relative amounts of exhaust flow particulates.

Beside the experimental investigation on the effect of the pulsating flow on the combustion performance, a model based on the momentum equation has been developed and applied to solid inert particles in the gas flow of the Rijke-type pulsating combustor as shown in figure (14) [13], by using a computational scheme based on a fourth order Runge-Kutta method. For application of the model, two types of particles have been considered: coal ash and elutriated material from coal combustion. The behavior of coal-ash-type and coal elutriated material particles have been investigated in the hot flow of a Rijke pulsating combustor.

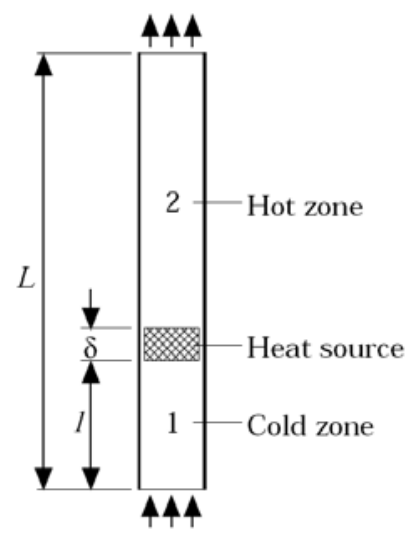

Fig. 14 Rijke tube with heat source. [13] 
The main results from the mathematical model [13] were as follows:

- The cut diameter, i. e., the diameter for which the particle starts to rise in the ascending gas flow of the combustor is the same for particles of the same density and dimensions in pulsating and non-pulsating flows of the same thermodynamic properties.

- The particle average velocity is higher in pulsating flows and, in consequence, the residence time for particles of the same density and dimensions is shorter in pulsating flows.

- Pulsating combustors tend to emit higher rates of particulates into the atmosphere.

- The particle velocity also possesses sinusoidal behavior in pulsating flows.

- The decrease in residence time became more significant when the particle diameter is close to the cut diameter, i.e., the diameter for which the particle starts to rise in the ascending gas flow of the combustor. The cut diameter is found to be independent of the type of regime, pulsating or non-pulsating.

Beside the effects of pulsating flow in enhancement the combustion process, pulsating flow has a great effect in the gas turbine engine cooling system.
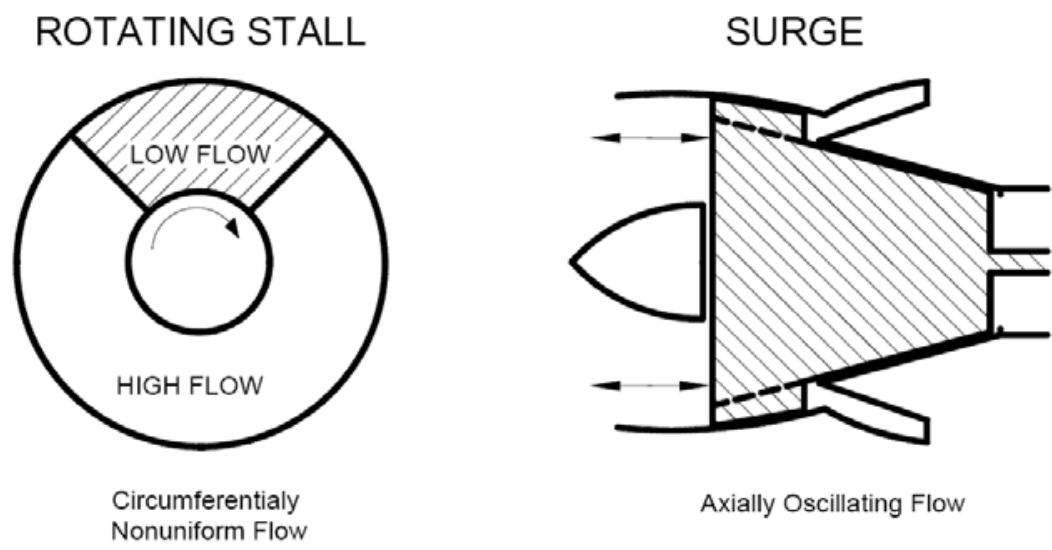

Fig. 15 Different forms of the compressor instability flow.

The effects of the compressor instabilities on the increase of turbine inlet temperature due to reduction of air flow rate, compressor instabilities (such as surge) will be the source of pulsating flow in the compression system, Figure (15). A group of researchers as [3], [5], [6], [14], [20] and [22] found that the pulsating flow is a suitable medium of the combustion process. The pulsating flow allows air and fuel to make a complete mixing before burning. These give the maximum output temperature from combustor which in turn increases the turbine thermal energy. But on the other hand, the pulsating flow has a great effect in the stability of pipes and ducts conveying air flow. The pulsating effect on the branching pipes flow was investigated by another group of researcher as [6] and [22]. This pulsating flow may block the branching flow due to nodding effect of the pulsating flow frequency. The effect of pulsating on the branching flow of the cooling passages will be discussed. The pulsating flow associated the compressor instabilities could be investigated assuming a simplified model of the gas turbine engine cooling passage. The compression system consists of a compressor working in an annular duct, which is connected to an exit plenum of much larger diameter. The discharge from the plenum is via a throttle of the plenum. The cooling passage is branched from the connected path between compressor and the plenum as shown in figure (16). Oscillations occurring in such a system are modeled in a manner analogous to those of a Helmholtz resonator and Greitzer model [3]. This model assumes that all the kinetic energy of 
the oscillation is associated with the motion of the fluid in the compressor and throttle ducts. Also, the potential energy is associated with the compression of the gases in the plenum. A schematic of the assumed model of the compression system, is shown in figure (16).

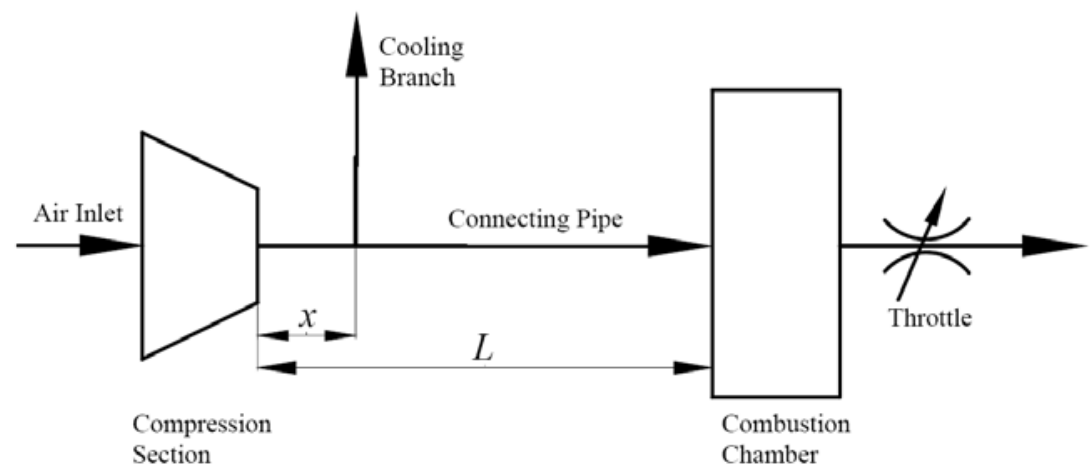

Fig. 16 Equivalent compression system [23]

Due to the instability of compressor operation when works in a surge zone, there are two pressure waves passing through the pipe connected compressor and plenum. These waves oscillate in the connected pipe and represent the surge mechanism. The wave propagates in the plenum path is assumed to have a dominated effect, figure (17). This case can be analyzed, by considering two transverse sinusoidal waves of the same amplitude, frequency and wavelength but traveling in opposite directions as formulated:

$$
\begin{aligned}
& \Delta p_{i}=\Delta p_{\text {max }} \sin (K x-\omega t+\phi) \\
& \Delta p_{r}=\Delta p_{\text {max }} \sin (K x+\omega t+\phi)
\end{aligned}
$$

where:

$\Delta p_{i} \ldots .$. . Pressure wave traveling in the $(+\mathrm{X})$ direction.

$\Delta p r$......Pressure wave traveling in the $(-\mathrm{X})$ direction.

To find out the frequency that can make a flow clogging at the inlet of cooling passage i.e. pressure difference is zero, the Eq. (8) has to be satisfied.

$\frac{\omega}{a} x=n \pi$

Figures (17) show that when the gas turbine engine compressor subjected a pulsating flow due to instabilities it could be harmfully effect on the engine cooling system and the whole engine performance. The engine surge produces pressure pulsation of frequency within 23-30 times of compressor rotor speed. This frequency is within $17-22 \mathrm{kHz}$ for the studied gas turbine engine. This high frequency could not be picked up by the available sensors [23]. Consequently, the surge problems can't be avoided by the control action. Pulsating flow frequencies depend on geometry of the gas turbine engine, compressor working conditions and position of cooling branch (x). 


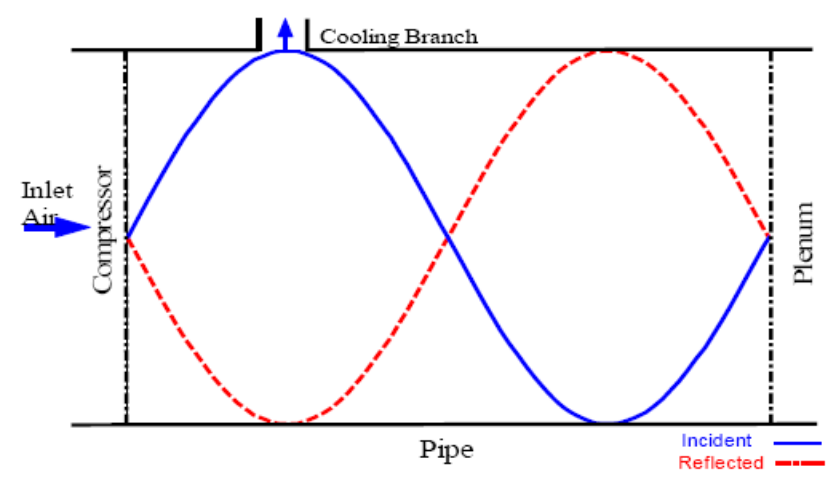

(a) Equivalent compression system with antinodes at inlet of cooling branch (design condition) [23]

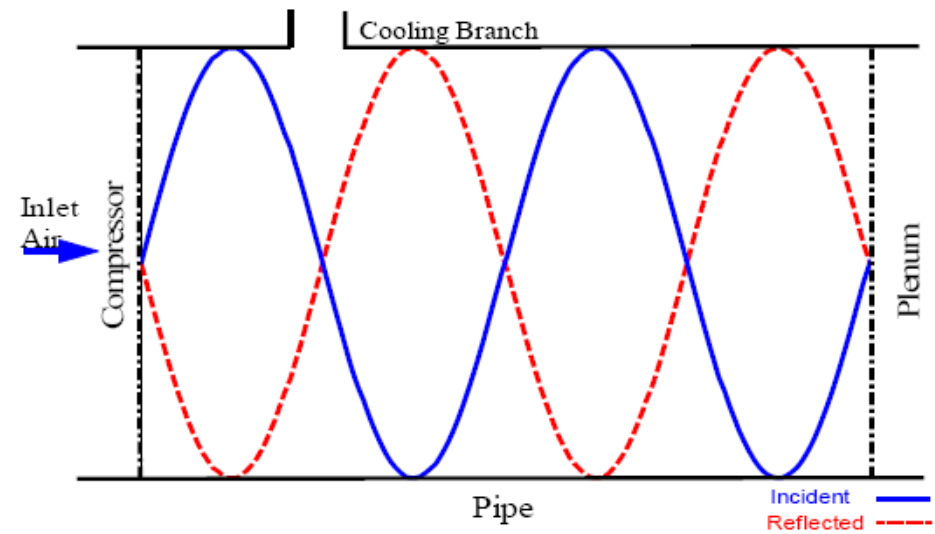

(b) Equivalent compression system with nodes at inlet of cooling branch[23]

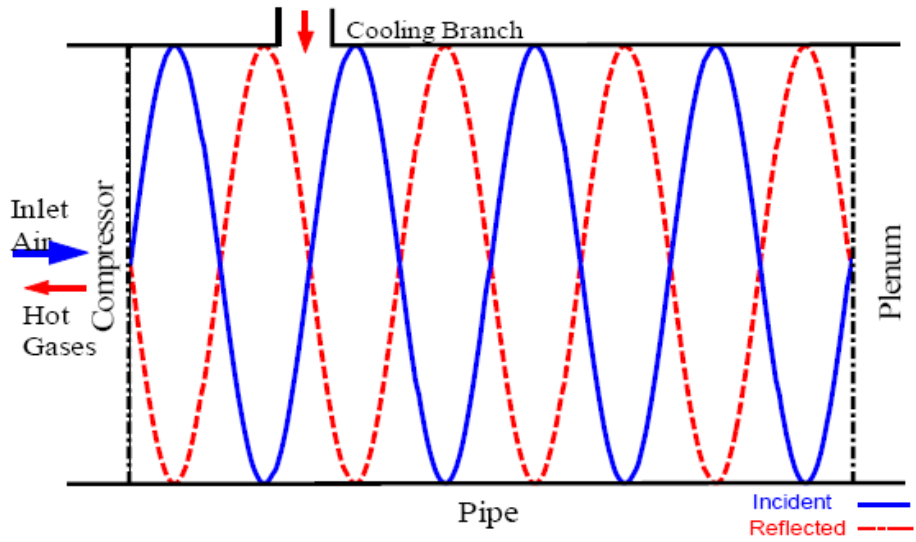

(c) Equivalent compression system with various nodding effect at inlet of cooling branch [23]

Fig. 17 Effect on cooling system 


\section{Effect of Pulsating Flow in the Noise:}

The flow through the exhaust pipe of a reciprocating combustion engine of an automobile is pulsating due to the opening and closing of the exhaust valve and involves the compression waves and the rarefaction waves. Such pressure waves move through the pipe, and reflect at some pipe elements and interact with each other. Thus, a complicated wave phenomenon is established. The compression wave propagating in the pipe often grows into a shock wave, depending on the rotational speed of the engine and the initial strength of the compression wave. The strength of the shock wave greatly influences the level of the noise emanating from the pipe end. The source of the noise has a complicated structure and the mechanism of the noise generation is not only related to the flow pattern throughout the pipe but also to that in the outside flow field. The jet discharged from the pipe into the atmosphere has subsonic and/or supersonic regimes depending on the flow pattern changing periodically in the pipe. In the subsonic regime of the jet, the turbulent vortex generated at the jet boundary is considered to be a main source of the noise. In the supersonic regime an under expanded jet is formed and the noise caused by instability of the jet is considered to be the main source of the noise. In practice, the shock wave is discharged from the pipe and subsequently the vortex ring forms at the edge of the pipe end, which is followed by the jet. They interact with each other, resulting in the emanation of noise. Since the interaction of the shock wave with the vortex ring and the jet boundary becomes a significant problem and of major interest to researchers from the viewpoint of acoustics and compressible fluid dynamics, many theoretical, numerical and experimental investigations have been carried out so far. Many papers treating this problem generally focus on the basic phenomena such as the interaction of the shock wave with the vortex ring and do not refer to their behavior in a real flow field. Such as, an experimental work has been carried out by simulating flow in an exhaust pipe attached to a four stroke engine $[17,18]$. The experimental has been carried out on the emission of exhaust noise from the open end of a pipe generated by the pulsating flow in the pipe. The pressure histories along the pipe, the exhaust noise and the visualized flow Field down-stream of the pipe end have been obtained. The frequency characteristics of the exhaust noise were also examined, using Wigner distribution (WD).

Figure (18) show the pressure histories at each station along the pipe measured at the rotation speed. The location of pressure transducers is shown by the solid points in the left side of this figure. The opening area change of the rotary valve is shown by the curve at the bottom of the figure. The abscissa denotes the time normalized by a period of one cycle of the rotary valve and the ordinate the pressure normalized by the ambient pressure. The process where the compression wave grows into a shock wave, which is considered to be one of the causes for the generation of metallic noise from the exhaust pipe, was reproduced in the present experiment. An attempt was made to clarify the mechanism of noise generation by relating the wave phenomena in the pipe with the exhaust noise.

- The relation between the pulsating flow and the exhaust noise, and the mechanism for the generation of the impulsive wave has been obtained. The following notes were drawn:

- The compression wave grows in the process of its propagation, and the pressure gradient becomes steep, shock wave being generated in the head of the compression wave toward the downstream end of the pipe.

- The impulsive wave is generated by the shock wave in the head of the compression wave when it arrives at the pipe end

- The magnitude of impulsive wave depends on the intensity of compression wave at the pipe end. 
- When the maximum intensity of compression wave is large, the directivity of the impulsive wave in the axial direction of the pipe becomes strong.

- This study didn't give an any kind of solutions for the metallic noise emitted from the exhaust pipe and the effect of pulsating flow on improvement of the performance of the exhaust pipe by diminish of the metallic noise.

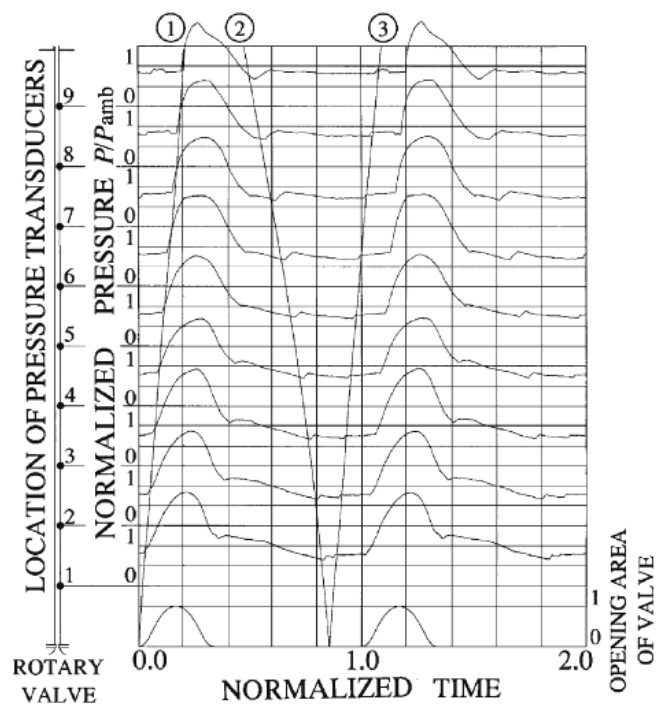

Fig. 18 Pressure histories at engine speed 2400 rpm. [17]

\section{5- Discussions}

- Pulsating flow can cause serious errors in certain types of flow meter such as the orifice plate, the turbine and vortex shedding flowmeters and the behavior of these types is well described in the following articles. The accurate measurement of dynamic differential pressure is vitally important in head class flowmeters such as the orifice plate, and this topic is discussed in detail by two different authors. If accurate measurement in pulsating flow cannot be guaranteed it may be necessary to damp the pulsation using acoustic filters. The performance of such filters is discussed in another article presented in this special issue.

- There are many meter types that have not been covered here because their behavior in pulsating flow is still unknown. In some cases, such as fluidic oscillators, significant pulsation effects are expected, whereas in other cases, including electromagnetic, ultrasonic time of- flight and Coriolis mass flow meters relatively small effects are likely. Experimental work is still required to determine how significant these effects are.

$>$ The pulsation frequencies which can be measured by a meter are restricted to less than approximately one quarter of the drive frequency. The use of overlapping data blocks can improve the detailed resolution of the waveform

$>$ The maximum pulsation error in a turbine meter is twice that produced in an orifice meter for the same amplitude pulsation

$>$ The effect of pulsating flow on the gas turbine engine was reviewed, from point of view of its effect on combustion and flow branching stability. The following are noticed: 
$>$ The frequency of the pulsating flow is not the major parameter in the enhancement of the combustion process. There are other parameters such as the diameter of the fuel particles.

$>$ Up to our knowledge, there is a lack in the dealing with the effect of the pulsating flow on the boundary layer.

$>$ Most researchers take into consideration, when analyzing the pulsating flow that the flow is in the fully developed shape. It is needed that to study the pulsating flow for flow not in fully developed shape.

- Effect of pulsating flow in flow system performance has been reviewed and the following are noticed:

The pulsating of air flow is favorable for the combustion process.

$>$ Pulsating flow leads to considerable reduction of the cooling air flow and hence an engine overheating problem may reach a very high value, especially in case of cooling air reduction. The cooling air flow rate is about $70 \%$ of total air flow rate.

$>$ Some compressor instabilities may results in surge problem. The pulsation of air flow is one of the results of the surge. The pulsation produces, in some cases zero or even negative pressure at inlet of the cooling air branch. The cooling air may be stopped or even reversed which results in catastrophic overheating of the engine.

$>$ The engine surge produces pressure pulsation of frequency within 23-30 times of compressor rotor speed. This frequency is within $17-22 \mathrm{kHz}$ for the studied gas turbine engine. This high frequency could not be picked up by the available sensors. Consequently, the surge problems cannot be avoided by the control action.

- Noise generation is the most unfavorable phenomenon associated the pulsating flow.

\section{References:}

[1] Mainardi et. al. (1977) [Pulsating duct flow in the presence of an orifice plate] international Journal of Mechanical Science, Vol. 19, pp. 533-546.

[2] J. S. Sabnis et. al. (1982) [Effect of oscillating flow on combustion rate of coal particles] journal of combustion and flame Vol.47 (157), pp 157-172

[3] Greitzer E. M. et. al., (1982), [A simple model for compressor stall cell propagation], Transaction of ASME, Journal of Engineering for Power, VOL. 104, pp. 170-176.

[4] T. Sasayama et. al. (1983) [A solid-state air flow sensor for automotive use] Journal of Sensors and Actuators Vol.4 (121), pp 121-128

[5] J. A. Carvalho, Jr et. al. (1987) [Combustion characteristics of un-pulverized coal under pulsating and non-pulsating conditions] Journal of FUEL, Vol. 66.

[6] W. M. Tien et. al. (1989) [Bifurcation behavior of nonlinear pipes conveying pulsating flow] Journal of Fluids and Structures Vol.3, pp 609-629.

[7] R. C. Mottram (1989) [Damping criteria for pulsating gas flow measurement] Journal of Flow Meas. Instruments, Vol I. pp 15-23

[8] W. M. Tien et. al. (1989) [Bifurcation behavior of nonlinear pipes conveying pulsating flow] Journal of Fluids and Structures Vol.3, pp 609-629.

[9] K. N. Atkinson (1992) [A software tool to calculate the over Registration error of a turbine meter in pulsating flow]. Journal of Fluids and Structures Vol.3, pp 700-714.

[10] R. C. Mottram et. al. (1992) [The influence of pulsating flows on orifice plate flowmeters] Journal of Flow Measurement Instruments, Vol. 3, No. 3, pp118-127. 
[11] L. Peizi et. Al (1993) [Noise radiation of a strongly pulsating tailpipe exhaust], Journal of Sound and Vibration, Vol. 167 (3), pp385-400.

[12] E. H. kansson and J. Delsing (1994) [Effects of pulsating flow on an ultrasonic gas Flowmeter], Flow Meas. Instrum., 1994 Vol.5, pp 93-101

[13] J. A. Carvalho JR (1995) [Behavior of solid particles in pulsating flows] Journal of Sound and Vibration Vol. 4, pp 581-593

[14] E. C. Mladint et. al. (1997) [Convective heat transfer to submerged pulsating jets] Journal of. Heat and Mass Transfer. Vol. 40, No. 14, pp. 321-330.

[15] N. Aretakis, et. al., (1997), [Wavelet analysis for gas turbine fault diagnostics], transaction of ASME, Journal of Engineering for Gas Turbine and Power, Vol. 119, pp. 870-876.

[16] H.J. Dane (1997) [Ultrasonic measurement of unsteady flow] Journal of Measurement, Vol. 8, pp 183-191.

[17] J. Higashiyama et. al. (1999) [Experimental study of exhaust noise generated by pulsating flow downstream of pipe end] Society of Automotive Engineers of Japan Vol. 20, pp 73-79

[18] M. Endo et. al. (2000) [Relation between the flow pattern downstream of duct and the noise] Society of Automotive Engineers of Japan JSAE, Vol. 21, pp 125-132.

[19] V. Skwarek et. al. (2001) [Measuring pulsating flow with ultrasound] Journal of Measurement, Vol. 29, pp 225-236.

[20] M. Dremin et. al., (2002), [Precursors of Stall and Surge Processes in Gas Turbines Revealed by Wavelet Analysis], Journal of Control Engineering Practice, Vol. 10, pp. 599-604.

[21] S. J. Lee et. al. (2003) [Quantitative analysis of flow inside the accumulator of a rotary compressor] International Journal of Refrigeration, Vol. 26, pp 321-327

[22] D. Skamnakis et.al. (2005) [Flow stability analysis and excitation using pulsating jets] C. R. Mecanique Vol. 333, pp 628-635

[23] M.Metwally (2007) [Fluid power system controller at start/stop conditions], Ph. D. thesis, MTC. Cairo. 